\title{
Mass and decay properties of double heavy baryons with a phenomenological potential model
}

\author{
S. Rahmani ${ }^{\text {a }}$ H. Hassanabadi, H. Sobhani \\ Faculty of Physics and Nuclear Engineering, Shahrood University of Technology, P. O. Box: 3619995161-316, Shahrood, Iran
}

Received: 5 February 2020 / Accepted: 21 March 2020 / Published online: 8 April 2020

(C) The Author(s) 2020

\begin{abstract}
We consider baryons in a nonrelativistic quark model, find the wave function and masses of the baryons with two heavy quarks including $\Xi_{b c}(b c u), \Xi_{c c}(c c u)$, $\Xi_{b b}(b b u), \Omega_{b b}(b b s), \Omega_{b c}(b c s)$ and $\Omega_{c c}(c c s)$. We calculate magnetic moments of these baryons. Using transition magnetic moments, we obtain the radiative decay widths for double heavy baryons. Then we calculate some of the semileptonic decay widths of these baryons. Our results are compared with other theoretical predictions.
\end{abstract}

\section{Introduction}

The semileptonic decays of heavy baryons are a useful tool to extract the Cabibbo-Kobayashi-Maskawa (CKM) matrix elements. An interesting study that has been attracting for a long time is the double heavy baryons with two heavy quarks consisting of charm or bottom. QCD spectrum includes doubly and triply charmed baryons. SELEX Collaboration announced a signal for the doubly charmed baryon $\Xi_{c c}^{+}$in the charged decay mode $\Xi_{c c}^{+} \rightarrow \Lambda_{c}^{+} K^{-} \pi^{+}$. It was the first observation of doubly charmed baryon reported by the SELEX Collaboration at Fermilab [1,2]. The SELEX Collaboration later observed a signal for the doubly charmed baryon $\Xi_{c c}^{+}$in the decay modes $\Lambda_{c}^{+} K^{-} \pi^{+}$and $p D^{+} K^{-}$ and they reported $M_{\Xi_{c c}^{++}}=(3518.7 \pm 1.7) \mathrm{MeV}$ [3]. In 2017, the LHCb collaboration observed a highly significant structure in the $\Lambda_{c}^{+} K^{-} \pi^{+} \pi^{+}$mass spectrum in the protonproton collisions. They reported that the structure is consistent with the doubly charmed baryon $\Xi_{c c}^{++}$with the mass: $M_{\Xi_{c c}^{++}}=(3621.40 \pm 0.72 \pm 0.27 \pm 0.14) \mathrm{MeV}$ [4]. As there are more doubly heavy baryons may be confirmed and discovered experimentally in the future, the theoretical studies about them are essential. They need to study from the theoretical investigation based on quark models [5-13], Heavy Quark Spin Symmetry [14, 15], QCD Sum Rules [16-18], lat- tice QCD [19], Light-cone sum rules [20] and Bethe-Salpeter equation approach [21]. Brodsky et al. found that the large SELEX value of the isospin splitting of the $\Xi_{c c}$ states implies that double charm baryons are very compact [22]. Faessler et al. calculated form factors and decay rates of the semileptonic decays of the lowest-lying double heavy baryons in the relativistic three-quark model [12]. The consequences of the heavy diquark symmetry are used to find the mass spectra and the decay widths of double heavy baryons [23]. Martynenko calculated ground state triply and doubly heavy baryons in a relativistic three-quark model [6]. Guberina et al. considered doubly charmed baryons as a system containing a heavy cc-diquark and a light quark, similarly as in a heavy-light meson [24]. Masses, P-wave excitations, decay modes, lifetimes, and prospects for detection of the doubly heavy baryons have been studied by Karliner and Rosner [7]. Transition form factors between double-heavy baryons have been calculated using a manifestly Lorentz covariant constituent three-quark model [25]. In other recent studies, Mehen and Mohapatra computed the leading correction to the hyperfine splitting of the double heavy baryons in the framework of nonrelativistic QCD [26]. One of the most important references on the exclusive weak decays is [27], which pointed out the discovery channel of the observation of $\Xi_{c c}^{++} \rightarrow \Lambda_{c}^{+} K^{-} \pi^{+} \pi^{+}$and $\Xi_{c}^{+} \pi^{+}$. The theoretical studies of weak decays of doubly heavy baryons in the latest three years started from [27] and [28]. According to the factorization theorem, Gerasimov and Luchinsky studied exclusive weak decays of doubly heavy baryons [29]. Masses and magnetic moments of double heavy baryons were studied in the hyper central description of the three-body system [30,31]. Albertus et al calculated static properties and semileptonic decays for the ground state of double heavy baryons by variational ansatz wave functions [32]. Recently Li et al. computed mass spectra and wave functions of double heavy baryons using Bethe-Salpeter equations [33].

a e-mail: s.rahmani120@gmail.com (corresponding author) 
Table 1 Doubly heavy baryons with their quantum numbers considered in this study ( $S_{h}$ denotes the spin of the two heavy quarks.)

\begin{tabular}{lll}
\hline Baryon (quark content) & $S_{h}$ & $J^{P}$ \\
\hline$\Xi_{c c}(\mathrm{ccu}), \Omega_{c c}(\mathrm{ccs})$ & 1 & $\frac{1}{2}^{+}$ \\
$\Xi_{c c}^{*}(\mathrm{ccu}), \Omega_{c c}^{*}(\mathrm{ccs})$ & 1 & $\frac{3}{2}^{+}$ \\
$\Xi_{b b}(\mathrm{bbu}), \Omega_{b b}(\mathrm{bbs})$ & 1 & $\frac{1}{2}^{+}$ \\
$\Xi_{b b}^{*}(\mathrm{bbu}), \Omega_{b b}^{*}(\mathrm{bbs})$ & 1 & $\frac{3}{2}^{+}$ \\
$\Xi_{b c}(\mathrm{bcu}), \Omega_{b c}(\mathrm{bcs})$ & 1 & $\frac{1}{2}^{+}$ \\
$\Xi_{b c}^{*}(\mathrm{bcu}), \Omega_{b c}^{*}(\mathrm{bcs})$ & 1 & $\frac{3}{2}^{+}$ \\
\hline
\end{tabular}

We aim to study the double heavy baryons in a phenomenological potential quark model within QCD-inspired nonrelativistic formalism. As we deal with heavy baryons, the nonrelativistic formalism is an appropriate method. In the next section, we will present the wave function of baryons in the hyperspherical coordinates and calculate the masses of the baryons with two heavy quarks. In Table 1, we have shown the doubly heavy baryons with their quantum numbers considered in this study. In Sect. 3, the magnetic moments of some double heavy baryons are obtained. In Sect. 4, we present the radiative decay width of double heavy baryons. In Sect. 5, we study the semileptonic decay widths of double heavy baryons and calculate branching ratios of $\Xi_{b c}, \Xi_{b b}$ and $\Omega_{b c}$. Discussions on the paper are in Sect. 6 .

\section{Baryonic wave function}

We consider baryons as bound states of three quarks. The internal quark motions are described by two Jacobi vectors, $\rho$ and $\lambda$ as $[30,34]$

$\boldsymbol{\rho}=\frac{1}{\sqrt{2}}\left(\mathbf{r}_{1}-\mathbf{r}_{2}\right), \lambda=\sqrt{\frac{2}{3}}\left(\frac{m_{1} \mathbf{r}_{1}+m_{2} \mathbf{r}_{2}}{m_{1}+m_{2}}-\mathbf{r}_{3}\right)$.

in the center of mass frame $\left(R_{c . m} .=0\right)$. Instead of the variables $\rho$ and $\lambda$ one can use the hyperspherical coordinates which include the angels $\Omega_{\rho}=\left(\theta_{\rho}, \phi_{\rho}\right)$ and $\Omega_{\lambda}=\left(\theta_{\lambda}, \phi_{\lambda}\right)$. In the hyperspherical coordinates, the hyperradius $x$ and the hyperangle $\zeta$ are defined as

$x=\sqrt{\rho^{2}+\lambda^{2}}, \quad \zeta=\tan ^{-1}\left(\frac{\rho}{\lambda}\right)$

The Hamiltonian of the three-body system can be written as

$H=\frac{p_{\rho}^{2}}{2 m_{\rho}}+\frac{p_{\lambda}^{2}}{2 m_{\lambda}}+V(x)$

where the kinetic energy operator takes the form $(\hbar=c=1)$

$-\left(\frac{\Delta_{\rho}}{2 m_{\rho}}+\frac{\Delta_{\lambda}}{2 m_{\lambda}}\right)=-\frac{1}{2 \mu}\left(\frac{d^{2}}{d x^{2}}+\frac{5}{x} \frac{d}{d x}-\frac{L^{2}\left(\Omega_{\rho}, \Omega_{\lambda}, \zeta\right)}{x^{2}}\right)$ in terms of the angles of hyperspherical coordinates $\Omega_{\rho}=$ $\left(\theta_{\rho}, \phi_{\rho}\right)$ and $\Omega_{\lambda}=\left(\theta_{\lambda}, \phi_{\lambda}\right)$. We can write the relation of eigenfunction of $L^{2}$ as $[30,34]$

$$
\begin{array}{r}
L^{2}\left(\Omega_{\rho}, \Omega_{\lambda}, \zeta\right) Y_{[\gamma], l_{\rho}, l_{\lambda}}\left(\Omega_{\rho}, \Omega_{\lambda}, \zeta\right) \\
\quad=\gamma(\gamma+4) Y_{[\gamma], l_{\rho}, l_{\lambda}}\left(\Omega_{\rho}, \Omega_{\lambda}, \zeta\right)
\end{array}
$$

Here, the grand-angular momentum $\gamma=2 n+l_{\rho}+l_{\lambda}$ with $n=0,1, \ldots$ and $l_{\rho}, l_{\lambda}$ are the angular momenta associated with the $\rho$ and $\lambda$ variables. The terms $m_{\rho}, m_{\lambda}, \mu$ are defined in terms of the constituent quark masses $m_{1}, m_{2}$ and $m_{3}$ as

$m_{\rho}=\frac{2 m_{1} m_{2}}{m_{1}+m_{2}}, m_{\lambda}=\frac{3 m_{3}\left(m_{1}+m_{2}\right)}{2\left(m_{1}+m_{2}+m_{3}\right)}, \mu=\frac{2 m_{\rho} m_{\lambda}}{m_{\rho}+m_{\lambda}}$.

We choose the interaction of the system as exponential and hyper-Coulomb terms plus spin dependent as

$V(x)=-\frac{2}{3} \frac{\alpha_{s}}{x}+\alpha e^{-\beta x}+V_{0} \sum_{i<j} \frac{\mathbf{s}_{i} \cdot \mathbf{s}_{j}}{m_{i} m_{j}}$

where $\alpha=0.2 \mathrm{GeV}, \beta=1 \mathrm{GeV}$ and $V_{0}=0.01 .2 / 3$ is the colour factor for the baryon and the exponential term stands for confinement. Equation (7) expresses the one-gluon exchange Coulomb-type potential at short distance and a small range confinement term. The strong running coupling constant can be obtained using the relation [30]

$\alpha_{s}=\frac{\alpha_{s}\left(\mu_{0}\right)}{1+\frac{33-2 n_{f}}{12 \pi} \alpha_{s}\left(\mu_{0}\right) \ln \left(\frac{\mu}{\mu_{0}}\right)}$

where $\alpha_{s}\left(\mu_{0}=1 \mathrm{GeV}\right)=0.6$ is considered. The use of a hyperCoulomb interaction in baryon structure dates back to decades ago due to observations that the low-lying spectrum of hadrons has a hydrogenlike structure. We also need to add a confining term to satisfy the confinement concept. Different types of confinement terms such as linear, harmonic oscillator and exponential type have been considered by researchers to study the properties of hadrons [10,34-38]. Patel et al. studied double heavy baryons using $\frac{-\tau}{x}+\beta x^{p}$ [31]. We assume an exponential term that can be expanded in terms of power series of $x$ to confine the quarks. For the spin-dependent part, the third term of Eq. (7), we have used the considered relations in Refs. [39-42] as $\sum_{i<j} b_{i j} \mathbf{s}_{i} . \mathbf{s}_{j}$ (where $\mathbf{s}_{i}$ and $\mathbf{s}_{j}$ are the spin operators of the ith and jth quark, respectively and $b_{i j}$ is related to the $\left.\left(m_{i} m_{j}\right)^{-1}\right)$ for spin splittings of $S$-wave baryons and fitted the potential coefficients with the masses of heavy baryons by applying our approach. Through a simple variational approach, which is one of the most popular approaches to deal with quantum mechanical few-body problems, we solve the three-body problem. In this method, one can get a virtual solution with an appropriately chosen function space. Applying the hyper-Coulomb radial wave function is reasonably valid for the lower states since they are confined in the low $x$ region where the Columbic term is important [34]. 
Table 2 Masses of ground states of double heavy baryons (in $\mathrm{GeV}$ )

\begin{tabular}{llllll}
\hline Baryon & $a$ & Our, $J^{P}=\frac{1}{2}^{+}$ & Others, $J^{P}=\frac{1}{2}^{+}$ & Our, $J^{P}=\frac{3}{2}^{+}$ & Others, $J^{P}=\frac{3}{2}^{+}$ \\
\hline$\Omega_{b b}$ & 0.127 & 10.364 & $\begin{array}{l}10.359[9] ; 10.320[52] ; 10.273[19] ; \\
\end{array}$ & & $9.97[16] ; 10.447[53] ; 10.230[11]$ \\
$\Omega_{b c}$ & 0.117 & 6.958 & $6.75[16]$ & 6.372 & $10.308[19] ; 10.467[53] ; 10.500[57] ; 10.486[5]$ \\
$\Xi_{c c}$ & 0.094 & 3.396 & $3.519[54] ; 3.547[55] ; 3.676[5] ; 3.581[56] 3.434$ & $3.690[7] ; 3.675[11] ; 3.753[5]$ \\
$\Xi_{b b}$ & 0.106 & 10.215 & $10.197[14] ; 10.060[56] ; 10.314[53] ;$ & 10.227 & $10.236[14] ; 10.169[11] ; 10.367[5] ;$ \\
$\Xi_{b c}$ & 0.101 & 6.805 & $6.919[14] ; 6.72[16]$ & 6.830 & $6.986[14]$ \\
$\Omega_{c c}$ & 0.107 & 3.552 & $3.71[33]$ & 3.578 & $3.814[33] ; 3.724[58]$ \\
\hline
\end{tabular}

Table 3 Magnetic moments of double heavy baryons (in $\mu_{N}$ )

\begin{tabular}{lllll}
\hline Baryon & Our $J^{P}=\frac{1}{2}^{+}$ & Others $J^{P}=\frac{1}{2}^{+}$ & Our $J^{P}=\frac{3}{2}^{+}$ & Others $J^{P}=\frac{3}{2}^{+}$ \\
\hline$\Omega_{b b}$ & 0.522 & $0.16[59]$ & -0.775 & $-0.662[32] ;-0.703[42] ;-0.73[63]$ \\
$\Omega_{b c}$ & 0.991 & $0.49[59] ; 0.396[31] ; 0.399[42,60]$ & -0.307 & $-0.111[48] ;-0.274[42]$ \\
$\Omega_{c c}$ & 1.467 & $0.74[59] ; 0.783[31] ; 0.635[61]$ & 0.160 & $0.12[42,60]$ \\
$\Xi_{c c}$ & -1.067 & $-0.208[61]$ & 2.670 & $1.24[48] ; 1.06[60]$ \\
$\Xi_{b b}$ & -1.992 & $-0.742[32]$ & 1.737 & $0.82[48]$ \\
$\Xi_{b c}$ & -1.528 & $-0.76[62] ;-0.52[59]$ & 2.204 & $2.011[42,60] ; 1.414[63]$ \\
\hline
\end{tabular}

The trial hyper radial wave function is taken as the hyperCoulomb radial wave function given by [30,31,43-45]

$\psi_{n, \gamma}(a, x)=N_{n o r}(a x)^{\gamma} e^{-a x} L_{n}^{2 \gamma+4}(a x)$

where $a$ is the variation parameter. $N_{\text {nor }}$ denotes the normalization constant and $L_{n}^{2 \gamma+4}$ (ax) represents the Laguerre polynomial. We can find the variation parameter $a$ for double heavy baryons as we have shown in Table 2 . Then, hyperradial eigenfunctions and eigenvalues can be calculated. We can calculate masses of double heavy baryons as $[10,43]$

$M_{\text {baryon }}=m_{1}+m_{2}+m_{3}+\langle H\rangle$

We report the masses of double heavy baryons in Table 2. In the numerical evaluation we get $m_{s}=0.483 \mathrm{GeV}, m_{u}=$ $m_{d}=0.336 \mathrm{GeV}, m_{b}=4.950 \mathrm{GeV}$ and $m_{c}=1.550 \mathrm{GeV}$ $[34,43]$.

\section{Magnetic moments of double heavy baryons}

We use the wave functions of the previous section to study magnetic moments of doubly heavy baryons. The magnetic moment of a baryon can be calculated as $[31,35,46]$

$\mu_{B}=\sum_{i}\left\langle\psi_{s f}\left|\mu_{i} \sigma_{i}\right| \psi_{s f}\right\rangle$ where the quark composition constituting the baryonic state $[31,46]$ is

$\mu_{i}=\frac{e_{i}}{2 m_{i}^{e f f}}$

in terms of the effective mass $[31,46]$,

$m_{i}^{e f f}=m_{i}\left(1+\frac{\langle H\rangle}{\sum_{i} m_{i}}\right)$

which may have different values in baryonic systems due to the incorporating the kinetic and binding energies in the constituent quark mass. It takes care of the bound state effects including its internal motions and interactions. In Eq. (11), $e_{i}$ and $\sigma_{i}$ stand for charge and spin of the respective constituent quark corresponding to the spin flavor wave function $\psi_{s f}$ of the baryonic system $[31,46]$. The magnetic moments of double heavy baryons are computed using Eqs. (11)-(13) in terms of the nuclear magnetons of $J=\frac{1}{2}$ and $J=\frac{3}{2}$ states of the considered baryons in Table 3.

\section{Radiative transitions of double heavy baryons}

In recent years the radiative transitions of double heavy baryons have been computed in some approaches such as nonrelativistic constituent quark model [47], Bag model [48], relativistic three-quark model [8], diquark picture in a relativized quark model [11]. However there exist large disparities between the predicted values, so it is important to study 
Table 4 Radiative decay width for double heavy baryons (in keV)

\begin{tabular}{lll}
\hline Decay & Our & Others \\
\hline$\Gamma\left(\Xi_{c c}^{*} \rightarrow \Xi_{c c} \gamma\right)$ & 1.649 & $2.08[48] ; 3.90[11] ; 14.6[47]$ \\
$\Gamma\left(\Xi_{b b}^{*} \rightarrow \Xi_{b b} \gamma\right)$ & 0.022 & $0.022[48] ; 0.059[8]$ \\
$\Gamma\left(\Xi_{b c}^{*} \rightarrow \Xi_{b c} \gamma\right)$ & 0.316 & $0.612[48] ; 0.209[64]$ \\
$\Gamma\left(\Omega_{b b}^{*} \rightarrow \Omega_{b b} \gamma\right)$ & 0.001 & $0.04[11]$ \\
$\Gamma\left(\Omega_{b c}^{*} \rightarrow \Omega_{b c} \gamma\right)$ & 0.002 & $0.0031[64]$ \\
$\Gamma\left(\Omega_{c c}^{*} \rightarrow \Omega_{c c} \gamma\right)$ & 0.326 & $0.949[48]$ \\
\hline
\end{tabular}

the decay behaviors such as radiative transitions of double heavy baryons besides the mass spectra. In terms of transition magnetic moments $\mu\left(B^{*} \leftrightarrow B\right)$ (in nuclear magnetons), the radiative decay width of double heavy baryons has the from [48]

$\Gamma=\frac{1}{137} \frac{\omega^{3}}{M_{P}^{2}} \frac{2}{2 J+1}\left(\frac{M_{B}}{M_{B^{*}}}\right) \mu^{2}\left(B^{*} \leftrightarrow B\right)$

where $M_{P}$ is the proton mass. $J$ and $M_{B^{*}}$ are the spin and the mass of decaying baryon [48]. $M_{B}$ is the mass of the baryon in its final state. The masses, magnetic moments and wave functions of doubly heavy baryons are adopted from our calculated values. In Eq. (14), the photon momentum is [48]

$\omega=\frac{M_{B^{*}}^{2}-M_{B}^{2}}{2 M_{B}}$

Transition magnetic moments can be obtained in terms of the constituting quarks $\left(\mu_{i}\right)$ of the initial and final states of the baryon. $\mu\left(B^{*} \leftrightarrow B\right)$ can be computed using the spin flavor wave functions of initial and final states [35]

$\mu_{B^{*} \rightarrow B \gamma}=\sum_{i}\left\langle\psi_{s f}^{B}\left|\mu_{i} \sigma_{i}\right| \psi_{s f}^{B^{*}}\right\rangle$

Here the effective mass of quarks is [35]

$m_{i}^{e f f}=\sqrt{m_{i\left(B^{*}\right)}^{e f f} m_{i(B)}^{e f f}}$

By considering Eqs. (12), (13), (16) and (17), we calculate the radiative transition for double heavy baryons in Table 4 .

\section{Semileptonic decays of double heavy baryons}

Weak decay rates of doubly heavy baryons are one of the significant subjects in studying properties of these heavy baryons $[27,28]$. Semileptonic decay rates of doubly heavy baryons were studied using the relativistic quark model $[12,25]$, in the quark-diquark approximation [49], heavy quark spin symmetry relations $[14,32]$ and many other useful approaches. We need to have form factors. To study semilep- tonic decay width of doubly heavy baryons, we use the following relations according to Ref. [25]:

$$
\begin{aligned}
\Gamma_{\frac{1}{2} \rightarrow \frac{1}{2}}= & \frac{G_{F}^{2}\left|V_{b c}\right|^{2} M_{i}^{5} r^{4}}{12 \pi^{3}} \int_{1}^{w_{\max }} d w \sqrt{w^{2}-1}\left(l_{1}^{+}(w) \eta^{2}(w)\right. \\
& \left.+l_{1}^{-}(w) \eta^{2}(w)\right), \\
\Gamma_{\frac{1}{2} \rightarrow \frac{3}{2}}= & \frac{G_{F}^{2}\left|V_{b c}\right|^{2} M_{i}^{5} r^{4}}{12 \pi^{3}} \int_{1}^{w_{\max }} d w \sqrt{w^{2}-1} l_{2}(w) \eta^{2}(w), \\
\Gamma_{\frac{3}{2} \rightarrow \frac{1}{2}}= & \frac{G_{F}^{2}\left|V_{b c}\right|^{2} M_{i}^{5} r^{4}}{24 \pi^{3}} \int_{1}^{w_{\max }} d w \sqrt{w^{2}-1} l_{3}(w) \eta^{2}(w),
\end{aligned}
$$

which are related to semileptonic decay widths of doubly heavy baryons with states $1 / 2$ to $1 / 2,1 / 2$ to $3 / 2$ and also $3 / 2$ to $1 / 2$ where $r=\frac{M_{f}}{M_{i}}, w_{\max }=\frac{M_{i}^{2}+M_{f}^{2}}{2 M_{i} M_{f}}$ and we have

$l_{1}^{ \pm}(w)=(w \mp 1)\left(3 w_{\max } \pm 1-2 w\right)$,

$l_{2}(w)=2(w+1)\left(w_{\max }-w+\frac{w^{2}-1}{6 r}\right)$,

$l_{3}(w)=2(w+1)\left(w_{\max }-w+\frac{\left(w^{2}-1\right) r}{6}\right)$

$V_{b c}$ denotes the Cabibbo-Kobayashi-Maskawa matrix element. We have taken $V_{b c}=0.0422$ [50]. $G_{F}$ is the Fermi Coupling constant. Its value is $G_{F}=1.166 \times 10^{-5} \mathrm{GeV}^{-2}$. We have calculated the semileptonic decay widths in the heavy quark limit using the Isgur-Wise function $\eta(w)$. HQS limit is valid at and close to zero recoil and the expressions for the rates simplify [25]. In the near zero recoil limit all weak transition form factors between double heavy baryons can be expressed by a single universal function $\eta(w)$ which is a function of kinematic parameter $w$, the dot product of the four-velocities of the initial and final double-heavy baryon [25]. We have used a simple form for the IW function as [25]

$\eta(w)=\exp \left(-3(w-1) \frac{m_{c c}^{2}}{\Lambda_{B}^{2}}\right)$

with $m_{c c}=2 m_{c}=3.1 \mathrm{GeV}$ for the $b c \rightarrow c c$ transitions, $m_{b b}=2 m_{b}=9.9 \mathrm{GeV}$ for the $b b \rightarrow b c$ transitions. We have calculated the rates using two values of $\Lambda_{B}$ as 2.5 and $3.5 \mathrm{GeV}$ [25]. By using of our calculated masses in Table 2 and Eqs. (18)-(24), we can obtain the semileptonic decay widths of double heavy baryons. Table 5 shows the values of the semileptonic decay widths. Using the relation

$\operatorname{Br}\left(\Xi_{b c}\right)=\Gamma\left(\Xi_{b c}\right) \times \tau\left(\Xi_{b c}\right)$

and lifetimes $\tau\left(\Xi_{b b}\right)=370 \times 10^{-15} s, \tau\left(\Xi_{b c}\right)=244 \times$ $10^{-15} s$ [7] and $\tau\left(\Omega_{b c}\right)=220 \times 10^{-15} s$ [51], we can find 
Table 5 Semeileptonic decay width of double heavy baryons (in $10^{-14} \mathrm{GeV}$ )

\begin{tabular}{llll}
\hline Decay & Our $\left(\Lambda_{B}=2.5 \mathrm{GeV}\right)$ & Our $\left(\Lambda_{B}=3.5 \mathrm{GeV}\right)$ & Others \\
\hline$\Xi_{b c} \rightarrow \Xi_{c c} \ell \bar{v}_{\ell}$ & 3.01 & 5.07 & $8.87[65] ; 2.30[49]$ \\
$\Xi_{b b} \rightarrow \Xi_{b c} \ell \bar{v}_{\ell}$ & 0.49 & 1.37 & $28.5[66] ; 1.63[49] ; 0.86[25]$ \\
$\Xi_{b b}^{*} \rightarrow \Xi_{b c} \ell \bar{v}_{\ell}$ & 0.28 & 0.66 & $0.52[66] ; 0.28[49]$ \\
$\Xi_{b c}^{*} \rightarrow \Xi_{c c} \ell \bar{v}_{\ell}$ & 0.74 & 1.16 & $0.43[14] ; 27.5[66]$ \\
$\Xi_{b b} \rightarrow \Xi_{b c}^{*} \ell \bar{v}_{\ell}$ & 0.60 & 1.45 & $0.25[25]$ \\
$\Xi_{b c} \rightarrow \Xi_{c c}^{*} \ell \bar{v}_{\ell}$ & 1.98 & 3.32 & $1.39,2.13,0.64[25]$ \\
$\Omega_{b b} \rightarrow \Omega_{b c} \ell \bar{v}_{\ell}$ & 0.99 & 2.48 & $3.69[67]$ \\
$\Omega_{b c} \rightarrow \Omega_{c c} \ell \bar{v}_{\ell}$ & 3.28 & 5.39 & $3.94[67] ; 2.48[49] ; 1.88[25]$ \\
$\Omega_{b b}^{*} \rightarrow \Omega_{b c} \ell \bar{v}_{\ell}$ & 0.30 & 0.69 & $0.26[25]$ \\
$\Omega_{b c}^{*} \rightarrow \Omega_{c c} \ell \bar{v}_{\ell}$ & 0.80 & 1.23 & $0.59[25]$ \\
$\Omega_{b c} \rightarrow \Omega_{c c}^{*} \ell \bar{v}_{\ell}$ & 2.15 & 3.52 & $2.19,1.78,0.62[25]$ \\
$\Omega_{b b} \rightarrow \Omega_{b c}^{*} \ell \bar{v}_{\ell}$ & 0.63 & 1.53 & $1.32,0.57[25]$ \\
\hline
\end{tabular}

Table 6 Branching fractions of semileptonic decay widths for double heavy baryons

\begin{tabular}{llll}
\hline Process & Our $\left(\Lambda_{B}=2.5 \mathrm{GeV}\right)$ & Our $\left(\Lambda_{B}=3.5 \mathrm{GeV}\right)$ & Ref. [67] \\
\hline$\Xi_{b c} \rightarrow \Xi_{c c} \ell \bar{v}_{\ell}$ & $1.11 \times 10^{-2}$ & $1.88 \times 10^{-2}$ & $1.67 \times 10^{-2}$ \\
$\Xi_{b b} \rightarrow \Xi_{b c} \ell \bar{v}_{\ell}$ & $0.28 \times 10^{-2}$ & $0.77 \times 10^{-2}$ & $1.86 \times 10^{-2}$ \\
$\Omega_{b c} \rightarrow \Omega_{c c} \ell \bar{v}_{\ell}$ & $1.10 \times 10^{-2}$ & $1.80 \times 10^{-2}$ & $1.32 \times 10^{-2}$ \\
\hline
\end{tabular}

the branching fractions of double heavy baryons reported in Table 6.

\section{Results and discussion}

In summary, we have calculated some of the properties such as masses, magnetic moments, radiative transitions, semileptonic decay widths and branching fractions of double heavy baryons in the hyperspherical coordinates. We have used this coordinates due to its simplicity for studying the double heavy baryons. We have obtained the masses of double heavy baryons in the third and fifth columns of Table 2. Second column of Table 2 shows the variation parameter of the wave functions. We have compared our calculated masses with Refs. [5,7,9,12,14,16,19,49,52-57] in the fourth and sixth columns of Table 2 . In Table 3 , we have compared our obtained values for magnetic moments with Refs. [31,32,42,48,59-63]. In the third column of Table 4 we have listed our predicted values with Refs. [8, 12,47,48,64] for radiative transitions between the $J^{P}=\left(\frac{3}{2}\right)^{+}$and $J^{P}=\left(\frac{1}{2}\right)^{+}$ ground doubly charmed and bottom baryons. In the cases $J^{P}=\left(\frac{1}{2}\right)^{+}$ground states, only weak decays can happen. Our values for semileptonic decay widths are reported in in second and third columns of Table 5 comparing with other theoretical predictions $[14,25,49,65-67]$.

Our result in the case of $\Xi_{b c}$ is $\Gamma\left(\Xi_{b c}(b c u) \rightarrow \Xi_{c c}(c c u)+\right.$ $l \bar{\nu})=5.07 \times 10^{-14} \mathrm{GeV}$ which is in agreement with $(8.57) \times$
$10^{-14} \mathrm{GeV}$ [25]. Also our result $\left(1.37 \times 10^{-14} \mathrm{GeV}\right)$ is in agreement with $\left(1.63 \times 10^{-14} \mathrm{GeV}\right.$ [49]) reported for decay width of $\Xi_{b b} \rightarrow \Xi_{b c} \ell \bar{v}_{\ell}$

In our results there are three adjustable parameter $\alpha, \beta$ and $V_{0}$. These parameters shall be determined in the fitting procedure. Since we only access to experimental data of $\Xi_{c c}$ with $J=1 / 2$ configuration, these parameters are determined such that mass of this baryon was reproduced by 0.225 absolute error considering $\alpha=0.2, \beta=1$ and $V_{0}=0.01$. It should be noted that during the fitting procedure we have used $\hbar=c=1$. It is interesting to be pointed put mass splittings in $\mathrm{MeV}$ for doubly heavy $\Xi_{c c}$ baryon have been reported as $M_{\Xi_{c c}^{*}}-M_{\Xi_{c c}}=94_{-11}^{+5}$ [32]. Our calculations show $M_{\Xi_{c c}^{*}}-M_{\Xi_{c c}}=38 \mathrm{MeV}$. We have obtained other mass splittings as $M_{\Xi_{b b}^{*}}-M_{\Xi_{b b}}=12 \mathrm{MeV}, M_{\Xi_{b c}^{*}}-M_{\Xi_{b c}}=25$ $\mathrm{MeV}, M_{\Omega_{b b}^{*}}-M_{\Omega_{b b}}=8 \mathrm{MeV}, M_{\Omega_{b c}^{*}}-M_{\Omega_{b c}}=17 \mathrm{MeV}$ which are close to the $M_{\Xi_{b b}^{*}}-M_{\Xi_{b b}}=20 \pm 7 \mathrm{MeV}$ [68], $M_{\Xi_{b c}^{*}}-M_{\Xi_{b c}}=43 \pm 11 \mathrm{MeV}[68], M_{\Omega_{b b}^{*}}-M_{\Omega_{b b}}=19 \pm 5$ $\mathrm{MeV}$ [68] and $M_{\Omega_{b c}^{*}}-M_{\Omega_{b c}}=39 \pm 8 \mathrm{MeV}$ [68] respectively.

Our results for quantities masses, magnetic moments, radiative decay widths, semileptonic decay widths and branching ratios are in compatible with other predictions from reported references. The study of the double heavy quarks provides a new window for understanding the structure of these new baryons.

Acknowledgements The authors would like to thank the referee for his/her constructive criticisms and valuable suggestions. 
Data Availability Statement This manuscript has no associated data or the data will not be deposited. [Authors' comment: We have referred to the papers that we used their data in our work.]

Open Access This article is licensed under a Creative Commons Attribution 4.0 International License, which permits use, sharing, adaptation, distribution and reproduction in any medium or format, as long as you give appropriate credit to the original author(s) and the source, provide a link to the Creative Commons licence, and indicate if changes were made. The images or other third party material in this article are included in the article's Creative Commons licence, unless indicated otherwise in a credit line to the material. If material is not included in the article's Creative Commons licence and your intended use is not permitted by statutory regulation or exceeds the permitted use, you will need to obtain permission directly from the copyright holder. To view a copy of this licence, visit http://creativecomm ons.org/licenses/by/4.0/.

Funded by SCOAP ${ }^{3}$.

\section{References}

1. M. Mattson et al., SELEX Collaboration, Phys. Rev. Lett. 89, 112001 (2002)

2. M.A. Moinester et al., SELEX Collaboration, Czech. J. Phys. 53, B201 (2003)

3. A. Ocherashvili et al., Phys. Lett. B 628, 18-24 (2005)

4. R. Aaij et al., LHCb Collaboration, Phys. Rev. Lett. 119(11), 112001 (2017)

5. W. Roberts, M. Pervin, Int. J. Mod. Phys. A 23, 2817 (2008)

6. A.P. Martynenko, Phys. Lett. B 663, 317-321 (2008)

7. M. Karliner, J.L. Rosner, Phys. Rev. D 90, 094007 (2014)

8. T. Branz et al., Phys. Rev. D 81, 114036 (2010)

9. D. Ebert, R.N. Faustov, V.O. Galkin, A.P. Martynenko, Phys. Rev. D 66, 014008 (2002)

10. H. Hassanabadi, S. Rahmani, S. Zarrinkamar, Phys. Rev. D 90, 074024 (2014)

11. Q.F. Lü, K.L. Wang, L.Y. Xiao, X.H. Zhong, Phys. Rev. D 96, $114006(2017)$

12. A. Faessler, T. Gutsche, M.A. Ivanov, J.G. Körner, V.E. Lyubovitskij, Phys. Lett. B 518, 55-62 (2001)

13. H. Hassanabadi, S. Rahmani, Eur. Phys. J. Plus 131, 34 (2016)

14. E. Hernandez, J. Nieves, J.M. Verde-Velasco, Phys. Lett. B 663, 234 (2008)

15. J.M. Flynn, E. Hernández, J. Nieves, Phys. Rev. D 85, 014012 (2012)

16. T.M. Aliev, K. Azizi, M. Savc1, Nucl. Phys. A 895, 59-70 (2012)

17. K. Azizi, N. Er, Phys. Rev. D 100, 074004 (2019)

18. Z.G. Wang, Eur. Phys. J. C 78, 826 (2018)

19. Z.S. Brown, W. Detmold, S. Meinel, K. Orginos, Phys. Rev. D 9 , 094507 (2014)

20. Y.J. Shia, Y. Xing, Z.X. Zhao, Eur. Phys. J. C 79, 501 (2019)

21. Q.X. Yu, X.H. Guo, Nucl. Phys. B 947, 114727 (2019)

22. S.J. Brodsky, F.K. Guo, C. Hanhart, U.G. Meißner, Phys. Lett. B 698, 251-255 (2011)

23. B. Eakins, W. Roberts, Int. J. Mod. Phys. A 27, 1250153 (2012)

24. B. Guberina, B. Melic, H. Stefancic, Eur. Phys. J. C 13, 551-551 (2000)

25. A. Faessler, T. Gutsche, M.A. Ivanov, J.G. Korner, V.E. Lyubovitskij, Phys. Rev. D 80, 034025 (2009)

26. T. Mehen, A. Mohapatra, Phys. Rev. D 100, 076014 (2019)
27. F.S. Yu, H.Y. Jiang, R.H. Li, C.D. Lü, W. Wang, Z.X. Zhao, Chin. Phys. C 42, 051001 (2018). arXiv: 1703.09086

28. R.H. Li, C.D. Lü, W. Wang, F.S. Yu, Z.T. Zou, Phys. Lett. B 767, 232-235 (2017). arXiv:1701.03284

29. A.S. Gerasimov, A.V. Luchinsky, Phys. Rev. D 100, 073015 (2019)

30. B. Patel, A. Majethiya, P.C. Vinodkumar, Pramana J. Phys. 72, 679-688 (2009)

31. B. Patel, A.K. Rai, P.C. Vinodkumar, J. Phys. G Nucl. Part. Phys. 35, 065001 (2008)

32. C. Albertus, E. Hernández, J. Nieves, J.M. Verde-Velasco, Eur. Phys. J. A 32, 183 (2007). Erratum-ibid. A 36, 119 (2008)

33. Q. Li, C.H. Chang, S.X. Qin, G.L. Wang, Chin. Phys. C 44, 013102 (2020)

34. S. Rahmani, H. Hassanabadi, Few-Body Syst. 58, 150 (2017)

35. A. Majethiya, K. Thakkar, P.C. Vinodkumar, Chin. J. Phys. 54, 495-502 (2016)

36. G. Wagner, A.J. Buchmann, A. Faessler, J. Phys. G Nucl. Part. Phys. 26, 267-293 (2000)

37. K. Heikkila, N.A. Tornqvist, S. Ono, Phys. Rev. D 29, 110 (1984)

38. M.F.D. Ripelle, Phys. Lett. B 205, 97 (1988)

39. J.M. Richard, arXiv:hep-ph-9407224

40. J.M. Richard, arXiv: 1205.4326

41. R.C. Verma, M.P. Khanna, Prog. Theor. Phys. 77, 1019 (1987)

42. R. Dhir et al., Phys. Rev. D 88, 094002 (2013)

43. H. Hassanabadi, S. Rahmani, Few-Body Syst. 56, 691-696 (2015)

44. E. Santopinto, F. Iachello, M.M. Giannini, Eur. Phys. J. A 1, 307 (1998)

45. H. Ciftci, E. Ateser, H. Koru, J. Phys. A Math. Gen. 36, 3821-3828 (2003)

46. Z. Shah, A.K. Rai, Eur. Phys. J. C 77, 129 (2017)

47. L.Y. Xiao, K.L. Wang, Q.F. Lü, X.H. Zhong, S.L. Zhu, Phys. Rev. D 96, 094005 (2017)

48. A. Bernotas, V. Sǐmonis, Phys. Rev. D 87, 074016 (2013)

49. D. Ebert, R.N. Faustov, V.O. Galkin, A.P. Martynenko, Phys. Rev. D 70, 014018 (2004). Erratum-ibid. 77, 079903 (2008)

50. M. Tanabashi et al., Particle Data Group, Phys. Rev. D 98, 030001 (2018)

51. V.V. Kiselev, A.K. Likhoded, Usp. Fiz. Nauk 172, 497 (2002)

52. Z.G. Wang, Eur. Phys. J. C 68, 459 (2010)

53. T. Yoshida, E. Hiyama, A. Hosaka, M. Oka, K. Sadato, Phys. Rev. D 92, 114029 (2015)

54. C. Patrignani, Particle Data Group, Chin. Phys. C 40, 100001 (2016)

55. F. Giannuzzi, Phys. Rev. D 79(9) (2009)

56. V. Kovalenko, A. Puchkov, EPJ Web of Conferences, vol. 137, p. 13007 (2017)

57. T.M. Aliev, K. Azizi, M. Savci, J. Phys. G 40, 065003 (2013)

58. C. Alexandrou, V. Drach, K. Jansen, C. Kallidonis, G. Koutsou, Phys. Rev. D 90, 074501 (2014)

59. A. N. Gadaria, N. R. Soni, J. N. Pandya, Proceedings of the DAEBRNS Symp. on Nucl. Phys., vol. 61, p. 698 (2016)

60. R. Dhir, R.C. Verma, Eur. Phys. J. A 42, 243 (2009)

61. C. Albertus et al., Eur. Phys. J. A. 31, 691 (2007)

62. A. Faessler, T. Gutsche, M.A. Ivanov, J.G. Körner, V.E. Lyubovitskij, D. Nicmorus, K. Pumsa-ard, Phys. Rev. D 73, 094013 (2006)

63. A. Bernotas, V. Šimonis, arXiv:1209.2900v1

64. C. Albertus, E. Hernández, J. Nieves, Phys. Lett. B 690, 265 (2010)

65. A.I. Onishchenko, arXiv:hep-ph/0006295; arXiv:hep-ph/0006271

66. X.H. Guo, H.Y. Jin, X.-Q. Li, Phys. Rev. D 58, 114007 (1998)

67. W. Wang, F.S. Yu, Z.X. Zhao, Eur. Phys. J. C 77, 781 (2017)

68. N. Mathur, R. Lewis, R.M. Woloshyn, Phys. Rev. D 66, 014502 (2002) 